\title{
Control of internal and external noise in genetic regulatory networks
}

\author{
David Orrell, Hamid Bolouri \\ Institute for Systems Biology \\ 1441 North $34^{\text {th }}$ Street \\ Seattle, WA 98103 \\ U.S.A.
}

Corresponding author: D. Orrell dorrell@ systemsbiology.org

Phone: (206) 7321334

Fax: (206) 7321262

\begin{abstract}
Positive and negative feedback loops, for example where a protein regulates its own transcription, play an important role in many genetic regulatory networks. Such systems will be subject to internal noise, which occurs due to the small number of molecules taking part in some reactions. This paper examines the effect of feedback loops on noise levels. Error growth techniques from nonlinear dynamics are used to estimate the variance of a system around a steady state attractor. It is shown that variablity due to intrinsic stochasticity is directly linked to the stability of the steady state, and therefore to the system's resistance to external perturbations. The methods are demonstrated for a number of simple systems, including a genetic switch with homo-dimerizing regulatory protein, and an oscillator.
\end{abstract}

Keywords : genetic regulation, stochastic, bifurcation, feedback 


\section{Introduction}

Cellular pathways are inherently nonlinear, and incorporate both positive and negative feedback loops. Positive feedback loops often cause molecular concentrations to act in a switch-like, all-or-none manner, as observed in both enzymatic interaction networks (e.g. the MAPK switch in Xenopus embryos (Ferrell Jr. and Machleder 1998)), and in genetic regulatory networks (e.g. the sea urchin endomesoderm network (Davidson, Rast et al. 2002)). Negative feedback loops can control the speed of response to intra and intercellular events (Rosenberg, Elowitz et al. 2002), regulate the concentration of a molecular species to a fixed level, or lead to oscillations. Oscillations can be either damped (e.g. the level of NF- $\kappa$ B in the mammalian TNF $\alpha-N F-\kappa$ B pathway (Hoffmann, Levchenko et al. 2002)) or long lasting, as in the cell cycle oscillator controlling cell cleavage timing in Xenopus embryos (Goldbeter 1991; Borisuk and Tyson 1998). Identifying and characterizing the design principles which underlie such feedback paths is essential to understanding sub-cellular systems (Csete and Doyle 2002).

Genetic networks can be modeled using ordinary differential equations (ODE's) (Bower and Bolouri 2001; Neves and Iyengar 2002), and the techniques of nonlinear dynamics used to determine stable, periodic, and chaotic attractors, as well as bifurcations between these states (Guckenheimer and Holmes 1983; Borisuk and Tyson 1998). However, when studied at the level of a single cell, biological systems are not only nonlinear, but highly stochastic. The number of molecules of a particular species taking part in a reaction within a single cell may be of the order of tens or less (McAdams and Arkin 1999; Thattai and Van Oudenaarden 2001). Reactions are therefore not a smooth, continuous process, but an inherently random event that is better modeled using stochastic techniques (McQuarrie, Jachimowski et al. 1964; Zheng and Ross 1991). In stochastic systems, there is no such thing as a stable point (except in the absence of reactants), or a perfectly periodic system. This complicates the understanding of system behavior.

There has been considerable research in recent years, both theoretical and experimental, into the effects of noise in genetic networks. A number of stud ies have measured the 
amount of the random variation observed in the fluorescence distributions of a reporter protein, and used this to demonstrate that negative feedback suppresses noise (Becskei and Serrano 2000), or analyzed the effect of positive regulation in genetic switches (Becskei, Séraphin et al. 2001) and bistability (Isaacs, Hasty et al. 2003). Other studies have shown how noise is affected by translational and transcriptional efficiencies (Ozbudak, Thattai et al. 2002), and by internal and external contributions (Elowitz, Levine et al. 2002). The difference between eukaryotic and prokaryotic systems was explored in (Blake, Kaern et al. 2003). See for example (Paulsson 2004) and (Wall, Hlavacek et al. 2004) for useful reviews.

In this paper, we analyse stochastic noise from a nonlinear dynamics perspective, with the aim of providing fresh insights into the effects of noise on system dynamics. By combining the ODE and stochastic approaches, and using error growth techniques to approximate the variance of a system around a steady state equilibrium, the effect of auto-regulation on stochastic noise can be determined in a straightforward way from the model equations. The method is applied to a number of cases with positive and negative feedback, including a bistable switch and a genetic oscillator. Different methods of visualizing system behavior as a parameter varies are explored, which correspond to the bifurcation diagrams used in deterministic systems. We also discuss "design principles" which may underlie genetic networks, and illustrate the connection between variability due to internal stochasticity, and stability to external perturbations.

\section{Estimating stochastic noise}

In this section, we present a formula to estimate the variance of a protein $X$ which regulates its own production. Suppose that the production and decay of $X$ is given by the reactions:

$$
\begin{array}{ll}
\text { Reaction } & \text { Rate } \\
\varphi \rightarrow X & a_{1}=k_{v} f(X) \\
X \rightarrow \varphi & a_{2}=k_{d} X
\end{array}
$$


where $\varphi$ denotes the empty set. The function $f(X)$ reflects the auto-regulation by $X$ of its own transcription, while $k_{v}$ is the maximum rate of production, and $k_{d}$ the rate of degradation.

When $X$ is at its steady state $X_{e q}$, the rate of production equals the rate of decay, so $k_{d} X_{e q}=k_{v} f\left(X_{e q}\right)$. In the case of a stochastic simulation, however, $X$ will be continuously perturbed by random fluctuations. As discussed in the Appendix, the variance of $X$ around the steady state is given by

$V \cong h\left(X_{e q}\right) V_{0}$

where $V_{0}$ is the variance for the same protein level in the absence of regulation. If $X$ is the result of a Poisson process, its variance equals the mean, so $V_{0} \cong X_{e q}$. The term

$h\left(X_{e q}\right)=\left(1-\left.\frac{k_{v}}{k_{d}} \frac{\partial f}{\partial X}\right|_{X_{e q}}\right)^{-1}$

is a noise amplification factor. Using the equilibrium condition gives

$$
h\left(X_{e q}\right)=\left(1-\left(\frac{X}{f} \frac{\partial f}{\partial X}\right)_{X_{e q}}\right)^{-1}
$$

The estimate is obtained by linearizing the dynamics around the stable state; its accuracy therefore depends on a number of factors, but generally is lower near for example bifurcation points of the ODE where the linearization may not be valid. Estimates of the variance can also be obtained directly from the Master Equation (Van Kampen 1992) or a Langevin approach (Thattai and Van Oudenaarden 2002), however the error growth 
approach has a number of advantages. It can be used to derive analytical estimates for noise in simple systems, and so understand the dependence of noise on particular parameters; but can also be generalized to higher dimension systems, where calculating the variance through stochastic simulations can be extremely slow. The method has been incorporated into the program Dizzy (http://labs.systemsbiology.net/bolouri/Dizzy), which features both ODE and stochastic solvers, and is accessed using the “computeFluctuations" option.

If the production of $X$ is independent of $X$, then $\frac{\partial f}{\partial X}=0$ and $h\left(X_{e q}\right)=1$. This neutral situation will be the case when the production of $X$ is either unregulated, or the regulation is unaffected by $X$. If a genetic network increases the variance of $X$ above this level, so $h\left(X_{e q}\right)>1$, the network is noise enhancing in $X$, while if $h\left(X_{e q}\right)<1$ the network is noise reducing.

Two conclusions follow immediately from Eq. (3). Firstly, a network of the form of Eq. (1) is noise reducing if and only if $\frac{\partial f}{\partial X}<0$. Since this condition implies that the production of $X$ decreases with $X$, an equivalent statement is that a network with negative auto-regulation is noise reducing, and a network with positive auto-regulation is noise enhancing. This result is consistent with other theoretical and experimental works (Becskei and Serrano 2000; Thattai and Van Oudenaarden 2001).

Secondly, the formula provides a direct link between the variability due to internal stochasticity, and stability to external perturbations, as measured by their rate of decay. As shown in the Appendix, we can write

$h\left(X_{e q}\right)=k_{d} t_{d}$

where $t_{d}$ is the characteristic decay time of external perturbations. Reducing $t_{d}$ therefore both reduces internal variability and improves stability. Clearly there is a link between 
the decay rate $k_{d}$ and the decay time of perturbations; the trick of noise reduction is to reduce $t_{d}$ without increasing $k_{d}$. In the next sections, we give a number of specific examples of auto-regulated genetic networks which illustrate this point.

\section{Positive and negative auto-regulation}

A simple example of a network with negative regulation is one in which a protein $X$ represses its own formation. The reactions are:

$$
\begin{array}{ll}
\text { Reaction } & \text { Rate } \\
X+P \rightarrow X_{-} P & a_{1}=k_{b 1} X \cdot P \\
X_{-} P \rightarrow X+P & a_{2}=k_{b 2} X{ }_{-} P \\
P \rightarrow P+X & a_{3}=k_{v} P \\
X \rightarrow \varphi & a_{4}=k_{d} X
\end{array}
$$

The reactions describe the binding and unbinding of $X$ with a promoter $P$; the production of $X$, which occurs when the promoter is not bound with $X$; and the decay of $X$. The rate for each reaction is given by the corresponding term $a_{i}$, and depends on the rate parameters which we set to $k_{b 1}=5 \mathrm{~mol}^{-1} \mathrm{~h}^{-1}, k_{b 2}=100 \mathrm{~h}^{-1}$, and $k_{d}=1 \mathrm{~h}^{-1}$. Units are in terms of number of molecules per cell, rather than concentrations, and we assume a single molecule of $P$ in the bound or unbound state. Time is measured in hours, though it could equally well be some other unit. For simplicity, both transcription and translation have been modeled as a single step. As discussed in the Appendix, the intermediate step will introduce additional noise; however the degree of noise amplification due to autoregulation, as measured by $h\left(X_{e q}\right)$, is unchanged.

If the binding reactions are assumed to be at equilibrium, the ODE for this system can be written as

$\frac{d X}{d t}=\frac{k_{v} K}{K+X}-k_{d} X$ 
where $K=\frac{k_{b 2}}{k_{b 1}}$. For a particular value of $k_{v}$, the ODE will be attracted to a steady state which can be found by setting Eq. (7) to zero. A stochastic simulation, however, never achieves equilibrium, as shown in the left panel of Figure 1, which is a time series of $X$ for $k_{v}=150 \mathrm{~mol}^{-1} \mathrm{~h}^{-1}$. Calculations were performed with the program Dizzy, using the Gillespie algorithm (Gillespie 1976). Also shown are the mean, and the mean plus/minus one standard deviation.

While such plots are useful for seeing how the concentration varies with time, we are often more interested in visualizing how behavior changes as a function of a particular parameter. For example, in a real system the probability of transcription might depend on some factor such as the degree of phosphorylation of $X$, in which case $k_{v}$ could absorb this factor as discussed in (Smolen, Baxter et al. 1998). For ODE systems, a useful visualization tool is a plot of the equilibrium concentration of $X$ vs. $k_{v}$, which can be used to reveal for example bifurcation properties. This is shown by the black line in Figure 2A, which was obtained by setting Eq. (7) to zero at each value of $k_{v}$.

For the stochastic simulation, there is no single equilibrium point. An alternative method for visualizing the system's dependence on $k_{v}$ is illustrated by the color plots in the same panel. At each value of $k_{v}$, a 1000 hour stochastic simulation was performed, as in Figure 1. The value of $X$, which is an integer, was then binned, and the results displayed as a log density plot. Red indicates high density, while the blue background indicates zero density, so the plot shows the degree of spread in the range of $X$ introduced by stochasticity.

For this system the gradient of the fractional saturation function is

$$
\frac{\partial f}{\partial X}=-\frac{K}{\left(K+X_{e q}\right)^{2}}<0
$$

and the noise amplification is 
$h\left(X_{e q}\right)=\frac{K+X_{e q}}{K+2 X_{e q}}<1$

so the system is noise reducing with a maximum possible reduction in noise of $50 \%$ as $K$ approaches zero. Figure 2C compares the standard deviation of $X$ predicted using Eq. (3) to observed values from a stochastic simulation over 1000 time units. Also shown is the neutral case where the standard deviation equals $\sqrt{X_{e q}}$. The system shows a degree of noise reduction which is consistent with negative auto-regulation.

An example of positive auto-regulation is the system where transcription occurs when $X$ is bound to $P$, for which the ODE is:

$$
\frac{d X}{d t}=\frac{k_{v} X}{K+X}-k_{d} X+k_{a}
$$

The parameters are the same as before except that $k_{b 1}=1 \mathrm{~mol}^{-1} \mathrm{~h}^{-1}$, and $k_{a}=3 \mathrm{~mol} \mathrm{~h}^{-1}$ is a basal transcription rate, which is required for activation. The gradient of the fractional saturation function is

$\frac{\partial f}{\partial X}=\frac{K}{\left(K+X_{e q}\right)^{2}}>0$

which is identical to Eq. (8) except for the change of sign. The noise amplification factor can be calculated from Eq. (4).

The right panel of Figure 1 shows a time series of $X$. The parameter $k_{v}$ was set to 147 to give the same mean $X$ as in the left panel. Note the large increase in stochasticity relative to the previous case. Figure $2 \mathrm{~B}$ illustrates the behavior of this system as a function of $k_{v}$. The concentration of the product $X$ remains low until near $k_{v}{ }^{*}=k_{d} K$, at which the ODE undergoes a transcritical bifurcation (Alligood, Sauer et al. 1997). 
If the rate parameter $k_{v}$ were to depend on some factor such as the degree of phosphorylation of $X$, the system would behave like a genetic switch, with production of $X$ suddenly turning on around $k_{v}=100$. However, the effect of noise is so strong that the performance of the switch would be poor, with no clear distinction between the 'on' and 'off' states over this range of $k_{v}$ (clearly the impact of noise decreases for high levels of $k_{v}$ and resulting high protein concentrations, but the switch may need to operate with lower numbers of molecules). The predicted and observed noise amplification factor is shown in Figure 2D.

Comparing Eqs. (8) and (11), the difference between these two systems lies solely in the Jacobian. Since the Jacobian determines the decay time of perturbations, it is a measure of the stability of the steady state attractor. For 1-D systems such as these, it is possible to visualize some aspects of the nonlinear dynamics as illustrated in Figures $2 \mathrm{E}$ and $2 \mathrm{~F}$. The vertical axis is given by

$Y\left(X, k_{v}\right)=-\int_{0}^{X}\left(\frac{d X}{d t}\right) d X+Y_{m}\left(k_{v}\right)$.

where $Y_{m}\left(k_{v}\right)$ is the minimum value of $Y\left(X, k_{v}\right)$ for a given $k_{v}$. This has the property that the slope $\frac{d Y}{d X}$ in the $X$-direction is equal to $-\frac{d X}{d t}$, and the curvature at a steady state is equal to the Jacobian of the ODE. Thus steady states are points where the slope $\frac{d Y}{d X}$ is zero, which by the choice of $Y_{m}$ also satisfy $Y=0$. If the curvature is positive, the steady state is stable, while if it is negative the state is unstable. The strength of the stability depends on the degree of curvature. The resulting surface therefore allows visualization of the system dynamics and the basins of attraction (Alligood, Sauer et al. 1997) for each steady state. It is easy to see that the system with negative auto-regulation enjoys a higher 
degree of stability as $k_{v}$ increases. Furthermore, resistance to internal noise gives the added benefit of resistance to external noise.

To summarise the results of this section, we have seen through two simple examples how auto-regulation affects the degree of internal stochastic noise in genetic networks. Positive auto-regulation increases stochasticity, to the point where the performance of a genetic "switch" can be dominated by the effects of noise; while negative auto-regulation reduces noise (as measured by the variance) by as much as $50 \%$. The difference is due to the decay properties of the system, which can be visualized using the technique of Eq. (12), and is therefore linked directly to resistance to external perturbations, as measured by their rate of decay. Similar results can be obtained for more complicated transcriptional scenarios, for example where the gene contains multiple binding sites for the transcriptional proteins, by choosing an appropriate function $f(X)$.

From Eq. (11), one method to reduce noise amplification due to positive autoregulation is to simply reduce the binding dissociation constant $K$. The system then activates more quickly, since the ODE bifurcation point ${k_{v}}^{*}=k_{d} K$ is correspondingly lower. Thus the

system noise is directly linked to the value of $k_{v}{ }^{*}$, and it is impossible to have both a highly switchlike characteristic and low stochastic variability using positive autoregulation alone. In the next section, we consider another method to reduce variability while retaining switch-like characteristics, which actually increases the internal stochastic noise.

\section{Dimerization}

We now suppose that the protein $X$ binds with itself to form a homo-dimer, and the dimer then binds to DNA and enhances its own production (positive autoregulation). The resulting ODE is given by: 


$$
\begin{aligned}
& \frac{d X}{d t}=\frac{k_{v} X 2}{K+X 2}-2 k_{c 1} X^{2}+2 k_{c 2} X 2-k_{d} X+k_{a} \\
& \frac{d X 2}{d t}=k_{c 1} X^{2}-k_{c 2} X 2-k_{d} X 2
\end{aligned}
$$

The parameters $k_{c 1}=1 \mathrm{~mol}^{-1} \mathrm{~h}^{-1}, k_{c 2}=25 \mathrm{~h}^{-1}$ are the rates for dimerization and dedimerization of a protein $X$, where $X 2$ denotes the dimer. The binding parameters are $k_{b 1}=5 \mathrm{~mol}^{-1} \mathrm{~h}^{-1}, k_{b 2}=210 \mathrm{~h}^{-1}$, with $K=\frac{k_{b 2}}{k_{b 1}}$, while the decay and basal formation

parameters are $k_{d}=0.5 \mathrm{~h}^{-1}, k_{a}=0.5 \mathrm{molh}^{-1}$. The parameter $k_{v}\left(\mathrm{~h}^{-1}\right)$ is again adjustable. The structure is the same as that in (Smolen, Baxter et al. 1998) except that the decay of the dimer is included.

As shown by the solid line in Figure 3A, Eq. (13) has a bimodal nature with (for certain values of $k_{v}$ ) two stable steady states, and a sudden transition between the two. If $k_{v}$ is slowly increased from zero, the system jumps from a low state to a high state at the point that the lower steady state becomes unstable, around $k_{v}=140$; however if $k_{v}$ is reduced from a high value, the transition occurs when the high steady state becomes unstable, which is around $k_{v}=90$. The system therefore behaves as a hysteretic switch. Such all-ornothing responses have been observed on a cell-by-cell basis in Xenopus oocytes (Ferrell Jr. and Machleder 1998), and have been proposed as a mechanism by which a cell population can partition into different phenotypes (McAdams and Arkin 1997; Arkin, Ross et al. 1998).

Figure $3 \mathrm{~A}$ shows a density plot of the dimer $X 2$ as $k_{v}$ is slowly increased, while Figure 3B shows the same as $k_{v}$ is slowly decreased. The transition from low to high state, or vice versa, happens more rapidly than with the ODE, so the effect of hysteresis is lower. Comparison with Figure 2 shows that the equilibrium curves for the switches with and without dimerization are fairly similar, but the spread due to stochasticity in Figure 3C is substantially smaller. This is despite the fact that dimerization actually adds significantly 
to the internal stochasticity. These observations are in keeping with the results of (Bundschuh, Hayot et al. 2003).

How then does dimerization reduce noise? The reason is that noise is affected not just by the inherent stochasticity of the reactions, but, as mentioned above, by the decay characteristics of the system. The latter can be determined from an eigenvalue analysis of the Jacobian. For example, the Jacobian of Eqs. (13) at $k_{v}=150$ has two eigenvalues $\lambda_{1}=-197.6 \cong 4 k_{c 1} X_{e q}+k_{c 2}$ $\lambda_{2}=-0.30$

with corresponding eigenvectors $\mathbf{u}_{1}=[-0.89,0.47] \cong \frac{1}{\sqrt{5}}[-2,1], \mathbf{u}_{2}=[-0.281,-0.960]$. Both eigenvalues are negative, indicating stability, but there is a large difference in magnitude, which implies a fast and a slow time scale for decay. The first rate, with decay time $t_{d 1}=\frac{-1}{\lambda_{1}}$, corresponds to perturbations in the direction of the first eigenvector $\mathbf{u}_{1}$. This direction conserves the total number of protein molecules $X+2 \cdot X 2$, as occurs during the dimerization reaction. The second rate, with decay time $t_{d 2}=\frac{-1}{\lambda_{2}}$, corresponds to the direction of the second eigenvector $\mathbf{u}_{2}$. Because this is much slower, a perturbation in the direction $[1,0]$, as occurs during the formation or decay of $X$, or the direction $[0,1]$, as during the decay of $X 2$, will first equilibrate rapidly with the dimerization reaction, and then decay at the slower rate $t_{d 2}$. As shown schematically in Figure 4, the dimerization reduces such a perturbation by a factor $s=\frac{\sqrt{K_{c}^{2}+4 X_{e q}^{2}}}{K_{c}+4 X_{e q}}$. For given $X_{e q}$, the factor $s$ has a minimum of $\frac{1}{\sqrt{5}}$ when $K_{C}=X_{e q}$. Therefore, when stochastic effects perturb the system away from steady state, the effect of dimerization is to increase the rate of decay back towards the equilibrium. The enhanced stability of the system will again make it more resistant to externally generated noise. 
A rough analogy of dimerization might be a stiffening element in the roof of a building. Its own weight adds to the overall force (i.e. stochasticity) that needs to be supported, but if correctly positioned it can absorb the load of other components (the factor $s$ ) and result in a more efficient construction.

The improved performance of the switch with dimerization is actually a result of two factors: the reduction of stochastic noise due to the above effect, and the step change in $X$ and X2 which occurs near the ODE bifurcation point. A more relevant statistic than standard deviation might be the dimensionless ratio of standard deviation to the mean; with this measure, a sudden increase in the mean will lower the relative impact of noise. For the same reason, the performance can be further improved if the decay rate of the dimer is lower than that of the monomer as in Figure 3D, since this has the effect of increasing the mean dimer population.

The results of this section show that, by affecting the decay characteristics of the system, dimerization can be an important factor in the control of stochastic noise. It could therefore be viewed as a kind of "design principle" which might underlie genetic networks (de Atauri, Orrell et al. 2004; Wall, Hlavacek et al. 2004). In the next section, we consider a genetic system where noise might play a more positive role.

\section{An oscillator system}

A somewhat different example of a nonlinear system is the circadian oscillator described in (Gonze, Halloy et al. 2002). The system can again be written in terms of elementary reactions between a list of chemical species that includes enzymes, intermediate species, and bound and unbound forms of the gene. The result is a system of 24 reactions involving 19 chemical species. The number of gene copies is set to one, and the number

of other molecules is controlled by an adjustable parameter $\Omega$, which can be interpreted as a cell volume, so a concentration of $X \mathrm{nM}$ corresponds to $\Omega X$ molecules per cell. 
Since a system with fewer molecules tends to be more stochastic, the stochasticity varies inversely with $\Omega$. For this section, we consider two cases: a "high stochasticity" case with $\Omega=50$, and a "low stochasticity" case with $\Omega=200$.

The model can also be approximated in rate-law ODE form in five variables: $M_{P}$ is mRNA; $P_{0}, P_{1}$ and $P_{2}$ are cytosolic clock proteins; and $P_{N}$ is nuclear protein.

$$
\begin{aligned}
& \frac{d M_{p}}{d t}=\mathrm{v}_{s} \frac{K_{I}}{K_{I}+P_{N}}-\mathrm{v}_{m} \frac{M_{P}}{K_{m}+M_{P}} \\
& \frac{d P_{0}}{d t}=k_{s} M_{P}-\mathrm{v}_{1} \frac{P_{0}}{K_{1}+P_{0}}+\mathrm{v}_{2} \frac{P_{1}}{K_{2}+P_{1}} \\
& \frac{d P_{1}}{d t}=\mathrm{v}_{1} \frac{P_{0}}{K_{1}+P_{0}}-\mathrm{v}_{2} \frac{P_{1}}{K_{2}+P_{1}}-v_{3} \frac{P_{1}}{K_{3}+P_{1}}+\mathrm{v}_{4} \frac{P_{2}}{K_{4}+P_{2}} \\
& \frac{d P_{2}}{d t}=v_{3} \frac{P_{1}}{K_{3}+P_{1}}-\mathrm{v}_{4} \frac{P_{2}}{K_{4}+P_{2}}-\mathrm{v}_{d} \frac{P_{2}}{K_{d}+P_{2}}-k_{1} P_{2}+k_{2} P_{N} \\
& \frac{d P_{N}}{d t}=k_{1} P_{2}-k_{2} P_{N}
\end{aligned}
$$

Parameter values are:

$$
\begin{aligned}
& K_{I}=2 \mathrm{nM}, v_{s}=0.5 \mathrm{nM}, v_{m}=0.3 \mathrm{nM}, K_{m}=0.2 \mathrm{nMh}^{-1}, k_{s}=2 \mathrm{~h}^{-1}, \\
& v_{l}=6 \mathrm{nMh}^{-1}, K_{l}=1.5 \mathrm{nM}, v_{2}=3 \mathrm{nMh}^{-1}, K_{2}=1 \mathrm{nM}, \\
& v_{3}=6 \mathrm{nMh}^{-1}, K_{3}=1.5 \mathrm{nM}, v_{4}=3 \mathrm{nMh}^{-1}, K_{4}=2 \mathrm{nM}, \\
& v_{d}=1.5, K_{d}=0.1, k_{l}=2 \mathrm{nM}, k_{2}=1 \mathrm{nM} .
\end{aligned}
$$

Figure 5 shows a phase space plot, a time series plot, and a plot of power versus frequency for a stochastic simulation of the two cases with transcription rate $v=0.4$. Both cases show oscillatory behavior. Interestingly, however, the ODE system has a stable point for this value of $v$ (see also noted (Vilar, Kueh et al. 2002)).

Visualizing the behavior of nonlinear systems such as oscillators over a range of parameters can be difficult. One method is shown in Figure 6, which is a plot of the 
standard deviation of the species $M_{P}$ as a function of the transcription rate $v$, again over a range for which the ODE is stable. The results are compared with an estimate obtained using the 'computeFluctuations' option in Dizzy. Accuracy decreases for higher values of $v$, in part because the Jacobian becomes ill-conditioned near the bifurcation point; however the approximation remains useful and is considerably faster to compute than the full stochastic simulation.

While the standard deviation provides useful information, it does not indicate whether the power in the system is oscillatory or just random. An approach that better captures the system properties is the spectral bifurcation diagram (Orrell and Smith 2003), which shows the dominant oscillation frequencies as a parameter is varied. Figure 7 compares such diagrams for the ODE system and the stochastic simulations. The horizontal axis is the transcription rate $v$. The vertical axis is oscillation frequency, while the colour scale is the power at that frequency. For the ODE, all power is concentrated at the base circadian frequency and (to a smaller degree) its multiples, indicating that the oscillation is perfectly periodic, while the stochastic simulations spread the power over a range of frequencies.

At values of $v$ for which the ODE is periodic, the stochastic simulation adds noise but also power at the dominant frequency. In some respects, the stochastic simulation is more "robust" than the ODE model, in that it produces power over a greater range of frequencies; a desirable feature in oscillators (Barkai and Liebler 1999; Hasty, McMillen et al. 2001; Ma and Iglesias 2002). It can be viewed as an example of how biological systems exploit the presence of noise (Rao, Wolf et al. 2002). Of course this property is at the expense of perfect periodicity; but in any case biological oscillators must presumably rely on synchronizing effects (Höfer 1999; McMillen, Kopell et al. 2002) which would entrain the inherent oscillatory behavior to particular frequencies.

From this analysis, one difference between switches and oscillators is that what counts in the former is focusing of stochasticity into the desired switch-like behaviour, while in the latter stochasticity can actually add to the power of the oscillation. Even at parameter 
values for which the ODE is stable, the stochastic versions produce noisy oscillations with a power spike at the circadian frequency. It was seen in Section 4 that dimerization is a desirable feature in the genetic switch because it enhances stability. The purpose of an oscillator, however, is the opposite because it is intended to produce large swings in concentration. Dimerization would only retard this, because the system would then need to produce large changes in both the monomer and dimer concentrations. Therefore one might expect dimerization to play a larger role in genetic switches than oscillators.

\section{Conclusions}

In this paper we have combined the ODE and stochastic approaches to analyze the behavior of genetic systems. Our method for approximating the variance is easy to use, is freely available via the open-source program Dizzy, and can be used to derive simple formulas for the variance due to noise. By applying the method to a number of simple systems, it has been possible to make or confirm some specific statements about the effects of stochasticity:

- In agreement with the cited experimental results, auto-promotion was seen to increase noise, while auto-repression can decrease the variance by as much as $50 \%$.

- Control of internal noise is linked to stability to external perturbations through the decay characteristics. Therefore resistance to internal noise can also give resistance to external noise. Homeostatic systems will be resistant to both.

- Dimerization of regulatory proteins can reduce the effect of noise, not by reducing the inherent stochasticity of the reactions, but by altering the decay characteristics.

- Oscillators can exploit stochastic noise, by channeling it into oscillatory power.

Whatever the effect of stochasticity, it is clear that biological systems have evolved to control it and exploit it. One way to do this is by shaping the dynamic response by 
incorporating features such as negative feedback or dimerization. A better understanding of the interplay between stochasticity and nonlinearity should lead to improved comprehension of how these systems function; help deduce general principles which underlie their design; and motivate targeted experiments into the effects of stochastic noise. 


\section{Appendix: Estimating variance of stochastic models}

The variance of a stochastic model will depend both on the degree of stochasticity, and the nonlinear dynamics. We here show how it can be approximated using error growth techniques. Suppose that we have two models of a system: a stochastic simulation involving species $x_{n}$ for $n=1$ to $S$, such as that produced by the Gillespie algorithm (Gillespie 1976), and an ODE approximation. In general, the ODE has a reduced number of species, so for simplicity we assume the ODE variables are $x_{n}$ for $n=1$ to $R$ with $R<S$. We can view the difference between the stochastic simulation and the ODE model as an error produced by the different dynamics. The error growth can then be approximated by determining the propagated drift, which includes terms from the dynamical forcing error and the model dynamics (Orrell 2001; Orrell, Smith et al. 2001).

The first step is to approximate the forcing error of the stochastic model relative to an ODE representation. If the ODE is at a stable point, then an "error" of magnitude 1 occurs whenever a reaction happens that changes the ODE species by 1 molecule. Let

$a=\sum_{i=1}^{M} a_{i}$ be the sum of reaction rates over all $M$ possible reactions. Since at equilibrium the $M$ reactions are independent, the expected number of reactions of type $i$ over a sufficiently long time $T$ is $K_{i}=a_{i} T$.

Suppose that $c_{i}$ is the net change to the ODE species introduced by reaction $i$. For example, if the ODE only has species $X$ and $Y$ and a reaction $X+Y \rightarrow Z$ occurs, then the corresponding factor $c_{i}$ would be 2 . However if the reaction is $X+Z \rightarrow W$, then the factor $c_{i}$ would be 1 since the only change is to $X$; and if the reaction does not involve $X$ or $Y$, then $c_{i}=0$. Using a Euclidean metric, the difference between the states before and after the reaction is then $\sqrt{c_{i}}$. 
The drift at time $T$, evaluated numerically for a time step $\Delta t$, is given by a sum of short forecast errors $d(T)=\sum_{n=1}^{N} \mathbf{f}_{n}$, where $\mathbf{f}_{n}$ is the forecast error in a single time step (Orrell 2004). The drift represents the component of error (i.e. the difference between the stochastic and ODE representations) due to the model equations. A reaction of type $i$ over time $T$ implies a change of magnitude $\sqrt{c_{i}}$. Since the errors are stochastic, the total drift can be determined by summing the squares of the individual forecast errors from each reaction, so

$d(T)^{2}=\sum_{i=1}^{M} K_{i} c_{i}=\sum_{i=1}^{M} a_{i} T c_{i}=T a_{R}$

where $a_{R}=\sum_{i=1}^{M} a_{i} c_{i}$ is the sum of reaction rates weighted by the appropriate factor $c_{i}$. A further simplification arises by noting that the rate of change of each species $x_{n}$ is obtained from

$\frac{d x_{n}}{d t}=\sum_{i=1}^{M} w_{i} a_{i}$

where $w_{i}$ is equal to plus or minus the number of molecules of $x_{n}$ involved in the reaction, with the sign depending on whether $x_{n}$ is increased or decreased by the reaction. Since the scaling factor $c_{i}$ counts the total number of molecules involved in each reaction, it follows that

$\sum_{n=1}^{R} \sum_{i=1}^{M}\left|w_{i}\right| a_{i}=\sum_{i=1}^{M} c_{i} a_{i}=a_{R}$

The left hand side is just the sum of the absolute values of reaction rates in the ODE.

The expected forecast error at time $T=N \Delta t$, where $\Delta t$ is a discrete time step, is given by the propagated drift (Orrell 2002):

$\mathbf{d}_{p}(T)=\sum_{n=1}^{N} \mathbf{M}\left(T, t_{n}\right) \mathbf{f}_{n}$ 
Here $\mathbf{M}(T, t)=e^{\int_{t}^{T} \mathbf{J}(t) d t}=e^{(T-t) \mathbf{J}}$ is the linear propagator, and $\mathbf{J}$ is the Jacobian evaluated at the equilibrium, which is constant in time. Since the errors $\mathbf{f}_{n}$ are stochastic in nature, the expected root-mean-square (RMS) error $d_{p}(T)^{2}$ over an ensemble of runs will increase in a random walk fashion, so

$d_{p}(T)^{2} \cong \sum_{n=1}^{N}\left|\mathbf{M}\left(T, t_{n}\right) \mathbf{f}_{n}\right|^{2}$

The linear propagator reflects the growth (or shrinking) of small perturbations under the model dynamics, which depends on the linearized dynamics (Guckenheimer and Holmes 1983) near the attractor. The stability of a point can be analyzed by calculating the eigenvalues of the Jacobian (Ott 1993); if they are all negative, then the point is locally stable. For reactions with a linear decay, the propagator is of the form $e^{\left(-\frac{T-t_{k}}{t_{d}}\right)}$, where $t_{d}$ is the characteristic decay time. Since the forcing errors have magnitude 1 , the error is given by

$d_{p}(T)^{2} \cong \sum_{n=1}^{N} e^{\left(-2 \frac{T-t_{k}}{t_{d}}\right)}\left|\mathbf{f}_{n}\right|^{2}$

which asymptotes for large $T$ to the drift evaluated at half the decay time:

$d_{p}(T)^{2} \cong d\left(\frac{t_{d}}{2}\right)^{2}=a_{R} \frac{t_{d}}{2}$.

Thus the variance can be approximated by knowing only the reaction rates and the decay rate.

As an example, suppose that the production and decay of a protein $\mathrm{X}$ is given by the reactions:

$$
\begin{array}{ll}
\text { Reaction } & \text { Rate } \\
\varphi \rightarrow X & a_{1}=k_{v} f(X) \\
X \rightarrow \varphi & a_{2}=k_{d} X
\end{array}
$$


where $\varphi$ denotes the empty set. The corresponding ODE is

$\frac{d X}{d t}=a_{1}-a_{2}=k_{v} f(X)-k_{d} X$

so at equilibrium we have

$k_{d} X_{e q}=k_{v} f\left(X_{e q}\right)$

and the term $a_{R}$ is given by

$a_{R}=a_{1}+a_{2}=k_{v} f\left(X_{e q}\right)+k_{d} X_{e q}=2 k_{d} X_{e q}$.

The decay time can be calculated from the system Jacobian, which is

$J\left(X_{e q}\right)=\frac{\partial}{\partial X}\left(\frac{d X}{d t}\right)_{X_{e q}}=\left.k_{v} \frac{\partial f}{\partial X}\right|_{X_{e q}}-k_{d}$.

The decay time for small perturbations is then given by the negative inverse:

$t_{d}=\frac{-1}{J\left(X_{e q}\right)}=\frac{1}{k_{d}-\left.k_{v} \frac{\partial f}{\partial X}\right|_{X_{e q}}}$.

The variance is therefore given by

$V \cong a^{R} \frac{t_{d}}{2}=\frac{k_{d}}{k_{d}-\left.k_{v} \frac{\partial f}{\partial X}\right|_{X_{e q}}} X_{e q}=h\left(X_{e q}\right) X_{e q}$

where 
$h\left(X_{e q}\right)=\left(1-\left.\frac{k_{v}}{k_{d}} \frac{\partial f}{\partial X}\right|_{X_{e q}}\right)^{-1}$

is a noise amplification factor. Using Eq. (A10) gives

$h\left(X_{e q}\right)=\left(1-\left(\frac{X}{f} \frac{\partial f}{\partial X}\right)_{X_{e q}}\right)^{-1}$

which alternatively can be written in control coefficient notation as $h\left(X_{e q}\right)=\left(1-C_{X}^{f}\right)^{-1}$, or from Eq. (A13) as $h\left(X_{e q}\right)=k_{d} t_{d}$.

For simplicity, we have assumed that the protein $X$ is produced in a single step. If transcription and translation are modeled separately, the intermediate step will enhance the stochasticity in Eq. (A11) by a factor $\alpha$. If the decay rate of protein is much slower than that of mRNA, then $\alpha \cong 1+b$, where $b$ is the average number of proteins produced per mRNA lifetime (cf Thattai and Oudenaarden 2001); however the amplification of noise due to autoregulation remains the same. Thus we can write

$V \cong h\left(X_{e q}\right) V_{0}$

where $V_{0}$ is the variance for the same mean level of protein in the absence of autoregulation.

The method can be generalized to higher dimension systems. The decay time $t_{d}$ will depend on the type of perturbation, which can be accounted for by performing an eigenvalue analysis. Suppose the Jacobian $\mathbf{J}$ has eigenvalues $\lambda_{i}$ with corresponding eigenvectors $\mathbf{u}_{i}$. The drift can likewise be decomposed into the directions $\mathbf{u}_{i}$. The different components will then decay at rates given by $t_{i}=\frac{-1}{\lambda_{i}}$. For example, if 
transcription and translation are modeled as separate steps, a faster rate will typically correspond to the decay rate of mRNA, and a slower rate to the decay of protein. This method has been incorporated into the program Dizzy, and will presented in more detail in a separate paper. 


\section{References}

Alligood, K. T., T. D. Sauer, et al. (1997). Chaos: An Introduction to Dynamical Systems. New York, Springer-Verlag.

Arkin, A., J. Ross, et al. (1998). "Stochastic kinetic ana lysis of developmental pathway bifurcation in phage $\lambda$-infected Escherichia coli cells." Genetics 149: 1633-1648.

Barkai, N. and S. Liebler (1999). "Circadian clocks limited by noise." Nature 403: 267268.

Becskei, A., B. Séraphin, et al. (2001). "Positive feedback in eukaryotic gene networks: cell differentiation by graded to binary response conversion." EMBO 20: 2528-2535.

Becskei, A. and L. Serrano (2000). "Engineering stability in gene networks by autoregulation." Nature 405: 590-593.

Blake, W. J., M. Kaern, et al. (2003). "Noise in eukaryotic gene expression." Nature 422: 633-637.

Borisuk, M. T. and J. J. Tyson (1998). "Bifurcation analysis of a model of mitotic control in frog eggs." Journal of theoretical biology 195: 69-85.

Bower, J. M. and H. Bolouri, Eds. (2001). Computational modeling of genetic and biochemical networks, MIT Press.

Bundschuh, R., F. Hayot, et al. (2003). "The role of dimerization in noise reduction of simple genetic networks." J. Theor. Biol. 220: 261-269.

Csete, M. and J. Doyle (2002). "Reverse engineering of biological complexity." Science 295: 1664. 
Davidson, E., J. Rast, et al. (2002). "A provisional regulatory gene network for specification of endomesoderm in the sea urchin embryo." Dev Biol. 246: 162-190.

de Atauri, P., D. Orrell, et al. (2004). "Evolution of "design" principles in biochemical networks." IEE Sysems Biology 1.

Elowitz, M. B., A. J. Levine, et al. (2002). "Stochastic gene expression in a single cell." Science 297: 1183-1186.

Ferrell Jr., J. E. and E. M. Machleder (1998). "The biochemical basis of an all-or-none cell fate switch in Xenopus Oocytes." Science 280: 895-898.

Gillespie, D. T. (1976). "A general method for numerically simulating the stochastic time evolution of coupled chemical reactions." Journal of computational physics 22: 435-450.

Goldbeter, A. (1991). "A Minimal Cascade Model for the Mitotic Oscillator Involving Cyclin and cdc2 Kinase." PNAS 88: 9107-9111.

Gonze, D., J. Halloy, et al. (2002). "Robustness of circadian rhythms with respect to molecular noise." Proceedings of the National Academy of Science 99: 637-678.

Guckenheimer, J. and P. Holmes (1983). Nonlinear Oscillations, Dynamic Systems, and Bifurcations of Vector Fields. New York, Springer-Verlag.

Hasty, J., D. McMillen, et al. (2001). "Computational studies of gene regulatory networks: in numero molecular biology." Nature Reviews Genetics 2: 268-279.

Höfer, T. (1999). "Model of intercellular calcium oscillations in hepatocytes: synchronization of heterogeneous cells." Biophysical journal 77: 1244-1256. 
Hoffmann, A., A. Levchenko, et al. (2002). "The IkB-NF-KB signaling module: Temporal control and selective gene activation." Science 298(8).

Isaacs, F., J. Hasty, et al. (2003). "Prediction and measurement of an autoregulatory genetic module." PNAS 100: 7714-7719.

Ma, L. and P. A. Iglesias (2002). "Quantifying robustness of biochemical network models." BMC Bioinformatics $3: 38$.

McAdams, H. H. and A. Arkin (1997). "Stochastic mechanisms in gene expression" Proceedings of the National Academy of Science 94: 814-819.

McAdams, H. H. and A. Arkin (1999). "It's a noisy business! Genetic regulation at the nanomolar scale." Trends in genetics 15(2): 65-69.

McMillen, D., N. Kopell, et al. (2002). "Synchronizing genetic relaxation oscillators by intercell signalling." Proceedings of the National Academy of Science 99(2): 679-684.

McQuarrie, D. A., C. J. Jachimowski, et al. (1964). "Kinetics of small systems II." Journal of chemical physics 40: 2914-2921.

Neves, S. R. and R. Iyengar (2002). "Modeling of signaling networks." BioEssays 24: 1110-1117.

Orrell, D. (2001). Modelling nonlinear dynamical systems: chaos, error and uncertainty. Department of Mathematics, University of Oxford.

Orrell, D. (2002). "Role of the metric in forecast error growth: how chaotic is the weather?" Tellus 54A: 350-362. 
Orrell, D. (2004). "Filtering chaos: A technique to estimate dynamical and observational noise in nonlinear systems." International Journal of Chaos and Bifurcation.

Orrell, D. and L. A. Smith (2003). "Visualizing bifurcations in high-dimensional systems: The spectral bifurcation diagram." International Journal of Chaos and Bifurcations 13(10).

Orrell, D., L. A. Smith, et al. (2001). "Model error in weather forecasting." Nonlinear Processes in Geophysics 8: 357-371.

Ott, E. (1993). Chaos in dynamical systems, Cambridge University Press.

Ozbudak, E. M., M. Thattai, et al. (2002). "Regulation of noise in the expression of a single gene." Nature 31: 69-73.

Paulsson, J. (2004). "Summing up the noise in genetic networks." Nature 427.

Rao, C. V., D. M. Wolf, et al. (2002). "Control, exploitation and tolerance of intracellular noise." Nature 420: 231-237.

Rosenberg, N., M. Elowitz, et al. (2002). "Negative autoregulation speeds the response times of transcription networks." J. Mol. Biol. 323: 785-793.

Smolen, P., D. A. Baxter, et al. (1998). "Frequency selectivity, multistability, and oscillations emerge from models of genetic regulatory systems." American Journal of Physiology 274: C531-C542.

Thattai, M. and A. Van Oudenaarden (2001). "Intrinsic noise in gene regulatory networks." Proceedings of the National Academy of Science 98(15): 8614-8619. 
Thattai, M. and A. Van Oudenaarden (2002). "Attenuation of noise in ultrasensitive signaling cascades." Biophysical journal 82: 2943-2950.

Van Kampen, N. G. (1992). Stochastic processes in physics and chemistry. Amsterdam, North-Holland.

Vilar, J. M. G., H. Y. Kueh, et al. (2002). "Mechanisms of noise-resistance in genetic oscillators." Proceedings of the National Academy of Science 99(9): 5988-5992.

Wall, M. E., W. S. Hlavacek, et al. (2004). "Design of gene circuits: Lessons from bacteria." Nature Reviews Genetics 5: 34-42.

Zheng, Q. and J. Ross (1991). 'Comparison of deterministic and stochastic kinetics for nonlinear systems." Journal of chemical physics 94: 3644-3648. 
Figure legends

Figure 1. Plot of a stochastic simulation of the system in Eq. (6) with negative autoregulation (left panel), and system in Eq. (10) with positive auto-regulation (right panel). Parameters are as in the text, with $k_{v}=150$ in the former case, and $k_{v}=147$ (to give the same mean level) in the latter. Also shown are the mean, and the mean plus/minus one standard deviation. Simulation duration is deliberately long to get good statistics.

Figure 2. A and B show equilibrium values and $\log$ density plot of $X$ for the system with negative auto-regulation (left) and positive auto-regulation (right). Red indicates high density, blue low density. C and D compare the predicted standard deviation with the observed value. Also shown is the neutral case where there is no noise amplification. $\mathrm{E}$ and $\mathrm{F}$ show the basins of attraction for either system, as discussed in the text. The horizontal axis to the left is $X$, the axis to the right is $k_{v}$, so the view is from low to high $k_{v}$. Vertical axis $Y$ is defined by Eq. (12). Solid line is the equilibrium value of $X$ in the ODE. For high $k_{v}$ (back edge), the surface for negative regulation (left) has higher curvature than that on the right, implying greater stability.

Figure 3. A and B show density plot for $X$ as a function of the transcription rate $k_{v}$. The solid black line shows the stable equilibria for the ODE system, white line shows unstable equilibrium. In panel A, $k_{v}$ was started at zero and slowly increased, while in panel B $k_{v}$ was started at 170 and slowly decreased. There is a small effect due to hysteresis. Color scale indicates log of density. Panel C compares the standard deviation of the monomer $X$ and dimer $X 2$ with the neutral case as $k_{v}$ is increased. The improved performance of the system is due in part to noise reduction relative to the neutral case, and in part because of the jump to a higher steady state near the bifurcation point of the ODE. Panel D shows the density plot for the dimer $X 2$ when the decay rate of the dimer is halved, so that it decays more slowly than the monomer. 
Figure 4. Schematic diagram showing how homo-dimerization affects decay characteristics. Panel A shows decay of perturbations in $[X, X 2]$ phase space. Panels B and D compare estimated and measured decay as a function of time. Perturbations in the direction $[-2,1]$ corresponding to dimerization, i.e. constrained so that the total protein $X+2 \cdot X 2$ is unchanged, will decay rapidly at a rate given by $t_{d l}$ (black line, panel B). Perturbations in the direction $[1,0]$ decay rapidly in the direction $[-2,1]$ until the dimers are in equilibrium with the monomers (solid blue line, panels $\mathrm{A}$ and $\mathrm{D}$ ). The remaining perturbation, of magnitude $s$, then decays at the slow rate given by $t_{d l}$ (dashed blue line, panels A and D). The expression for $s$ (panel C) can be determined from the geometry. Perturbations in the direction [0,1] decay rapidly to a magnitude equal to $2 s$ (red lines, panels A and D). The horizontal time scales in B and D differ by a factor 1000 .

Figure 5. Oscillator behavior at transcription rate $v=0.4$, for which the ODE system has a stable point. Shown are the low ( $\Omega=200$ ) and high ( $\Omega=50$ ) stochasticity cases. Panels A and B show a phase diagram of $P_{N}$ versus $M_{p}$ (in nM). Trajectories initiated at the ODE stable point are perturbed in a stochastic fashion, and are drawn into an oscillatory behavior by the model dynamics. Panels $\mathrm{C}$ and $\mathrm{D}$ show a time series of $M_{p}, \mathrm{E}$ and $\mathrm{F}$ show the power spectrum with a clear spike near the circadian frequency of 1/24.

Figure 6. Standard deviation of $M_{p}$ (in $\mathrm{nM}$ ) as a function of transcription rate $v$ for the oscillator model. Shown are the high ( $\Omega=50)$ and low $(\Omega=200)$ stochasticity cases, as well as the estimated standard deviation, which scales as $\frac{1}{\sqrt{\Omega}}$. The accuracy of the estimate is better for the low stochasticity case, and decreases as $v$ approaches the bifurcation point and the Jacobian becomes ill-conditioned, but the method still provides a useful approximation over the range.

Figure 7. Spectral bifurcation diagrams for the ODE circadian oscillator (A), the stochastic simulation with low stochasticity (B), and high stochasticity (C). Horizontal axis is the transcription rate $v$, vertical axis is the $M_{p}$ frequency, colour is the log power at that frequency. The ODE shows power at higher values of $v$ only at a base frequency of 
about $1 / 24$ and its multiples, indicating periodicity. The stochastic simulations show power at a range of frequencies, with a peak near the circadian frequency, and extends to lower ranges of $v$. At several values of $v$, the stochastic trajectories drop down to lower frequencies. 


\section{Figures}
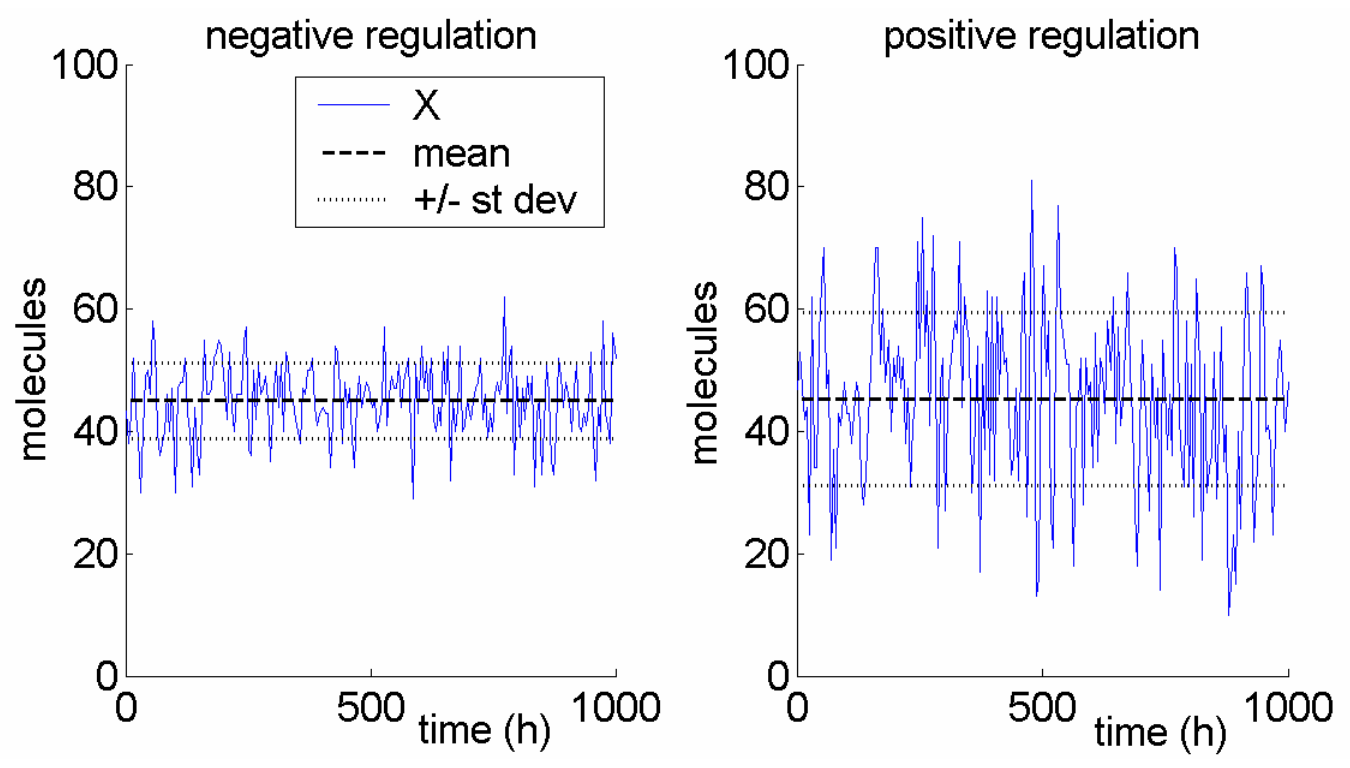

FIGURE 1 

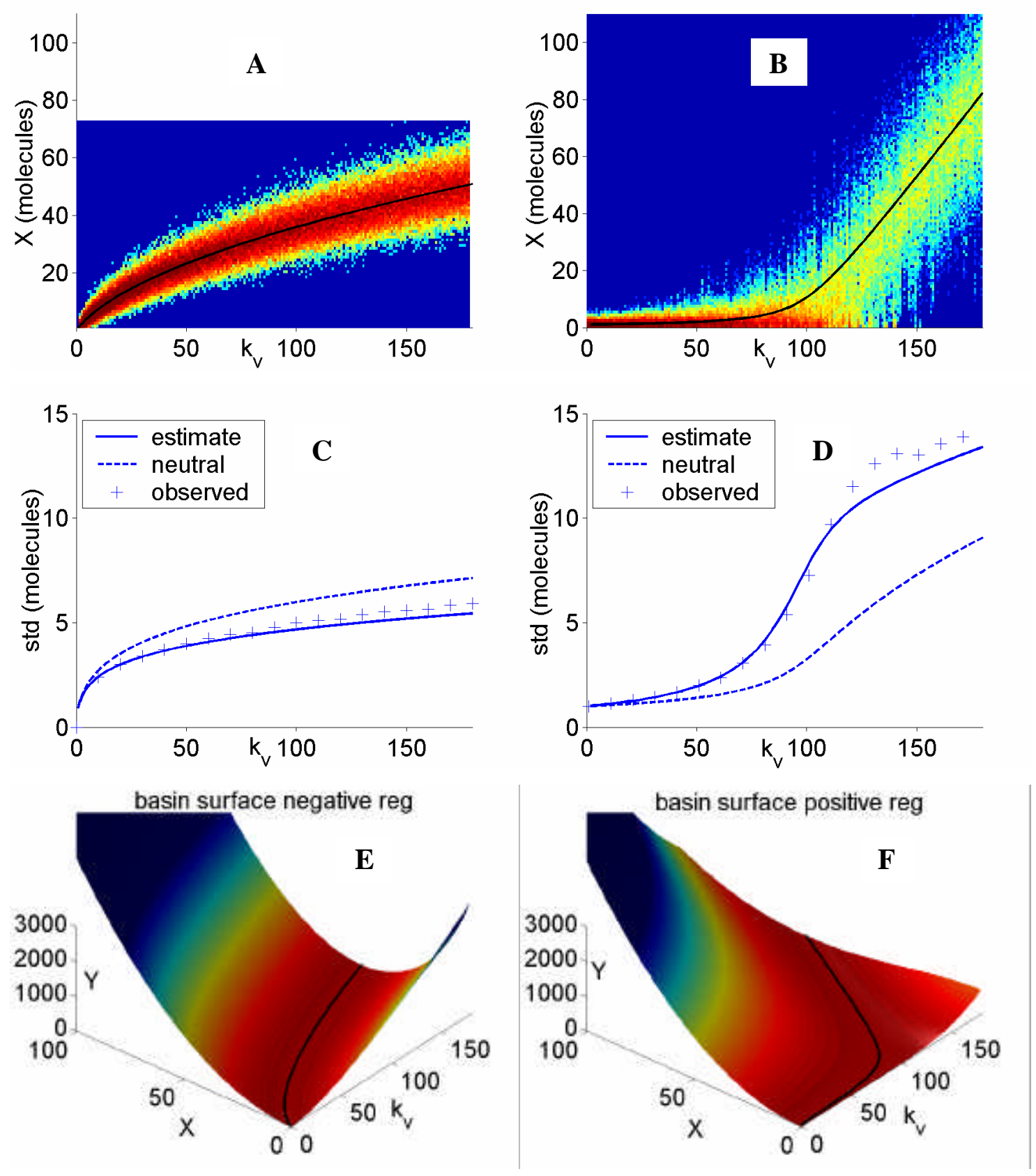

FIGURE 2 

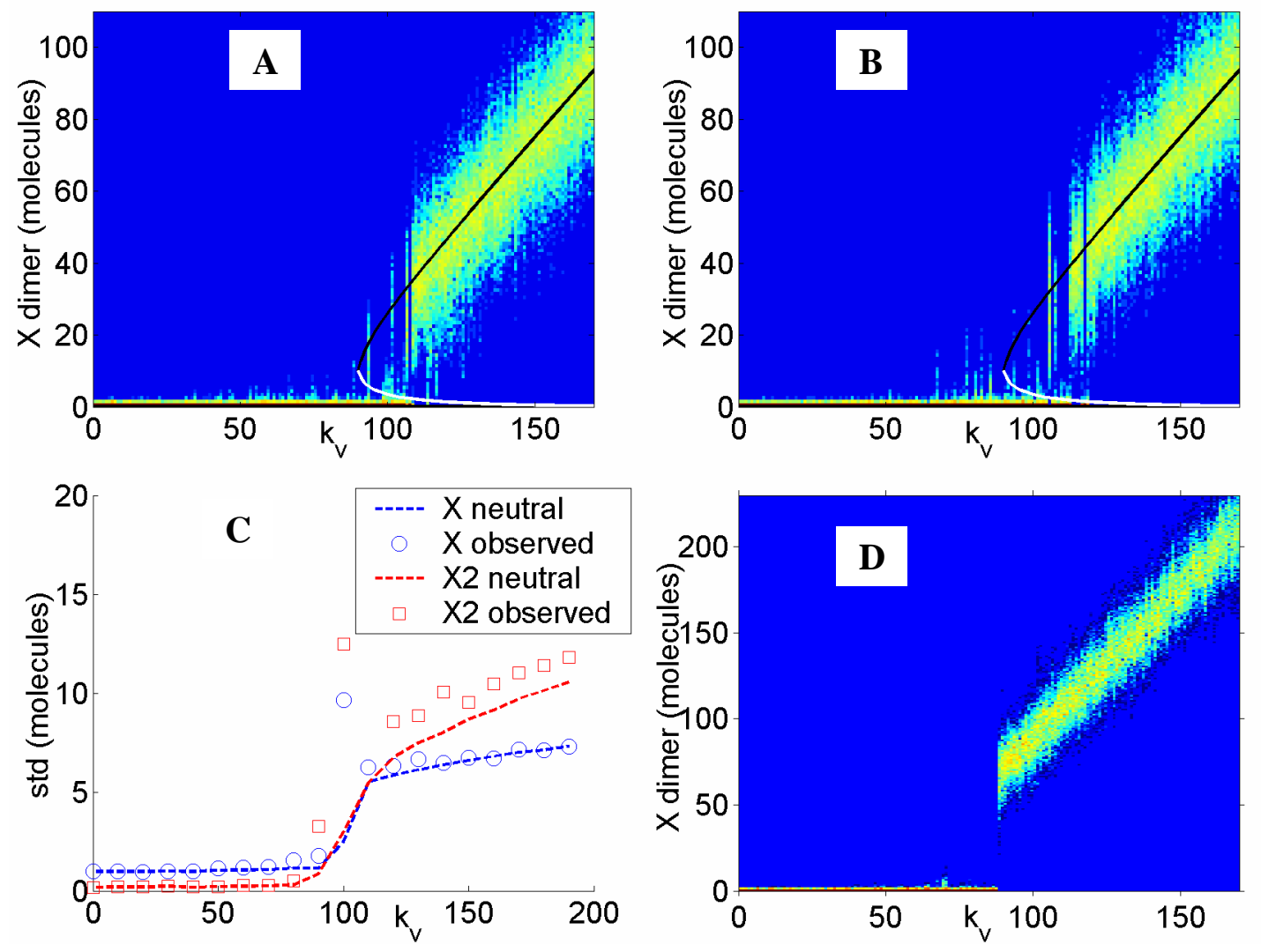

FIGURE 3 


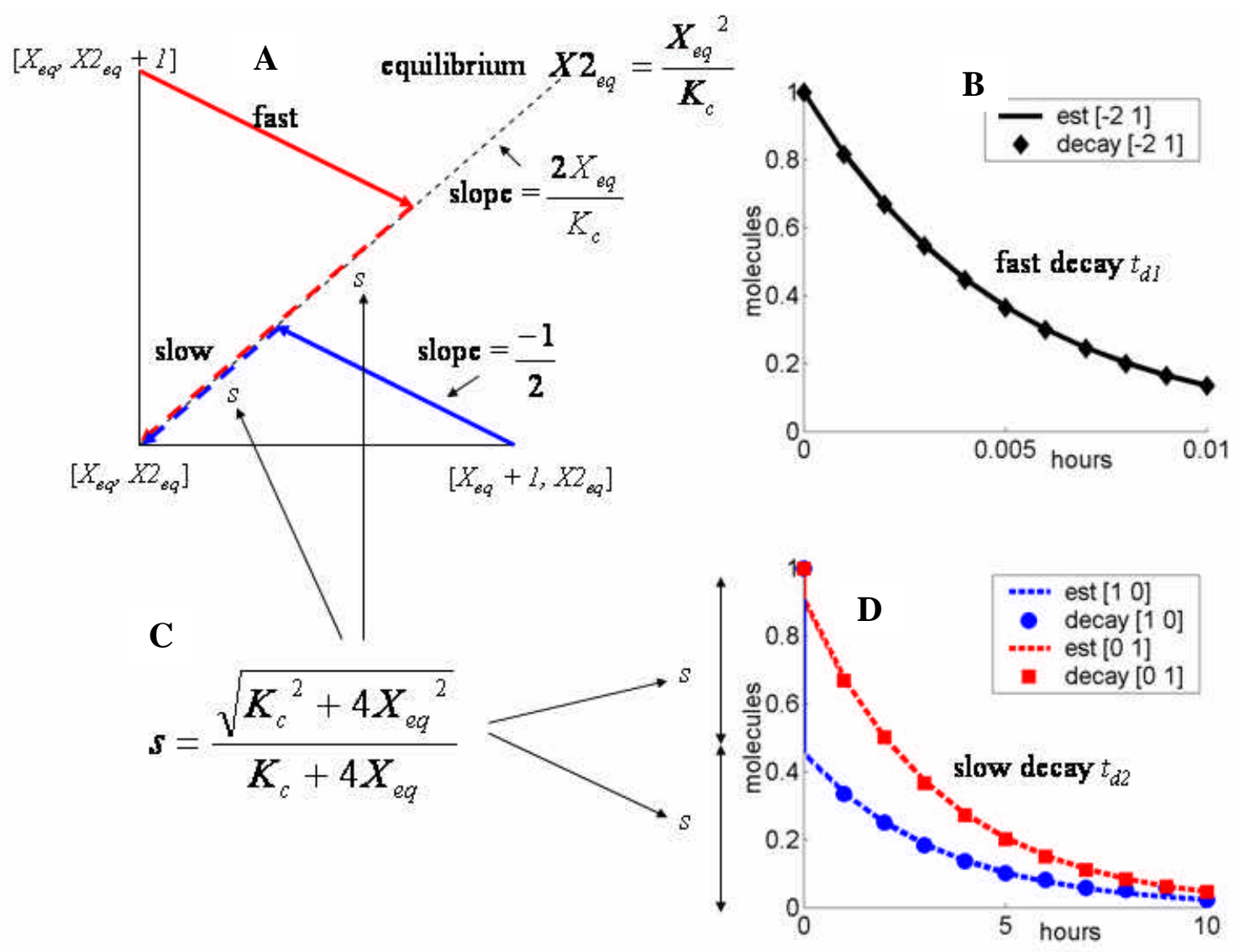

FIGURE 4 

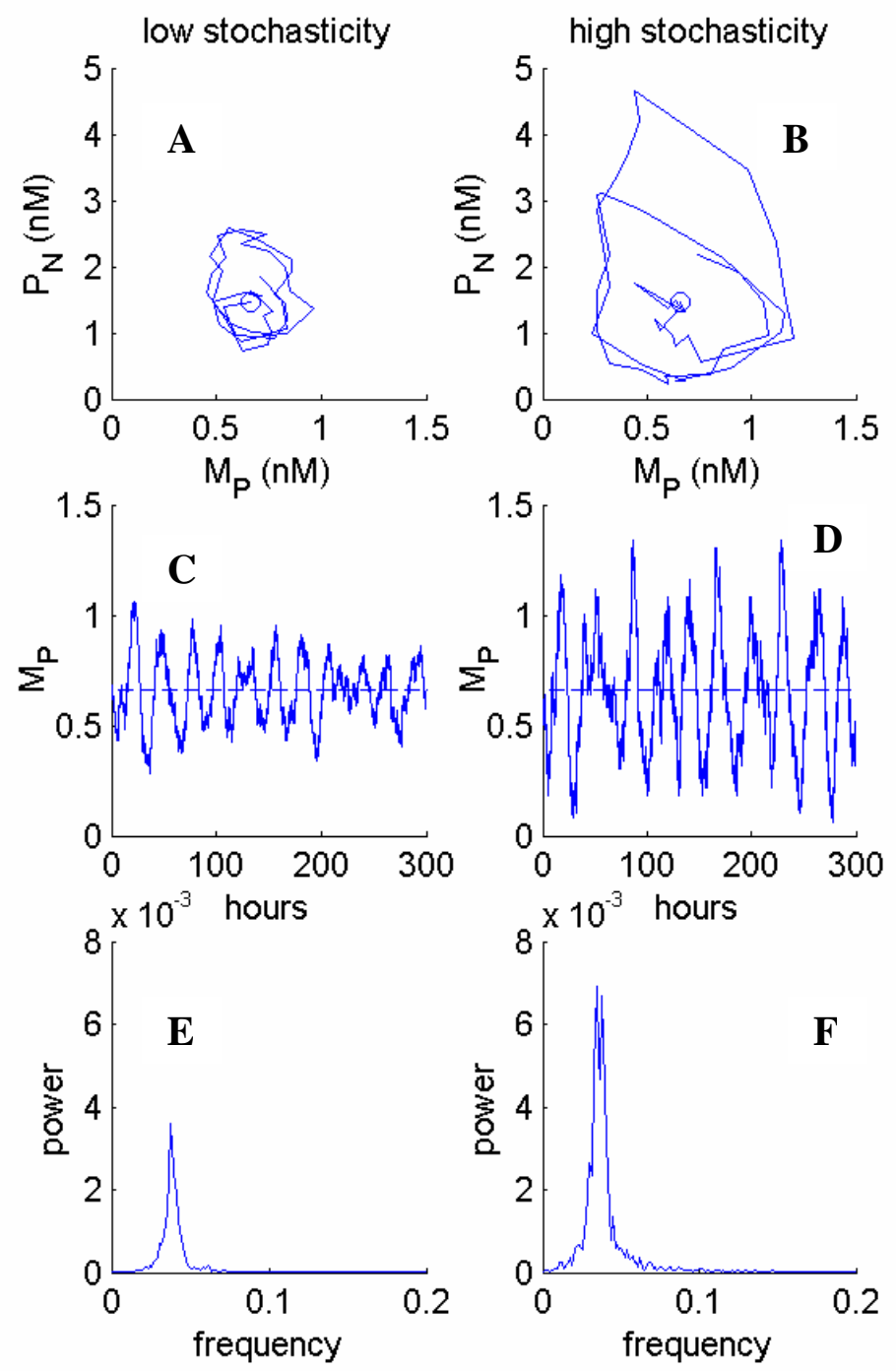

\section{FIGURE 5}




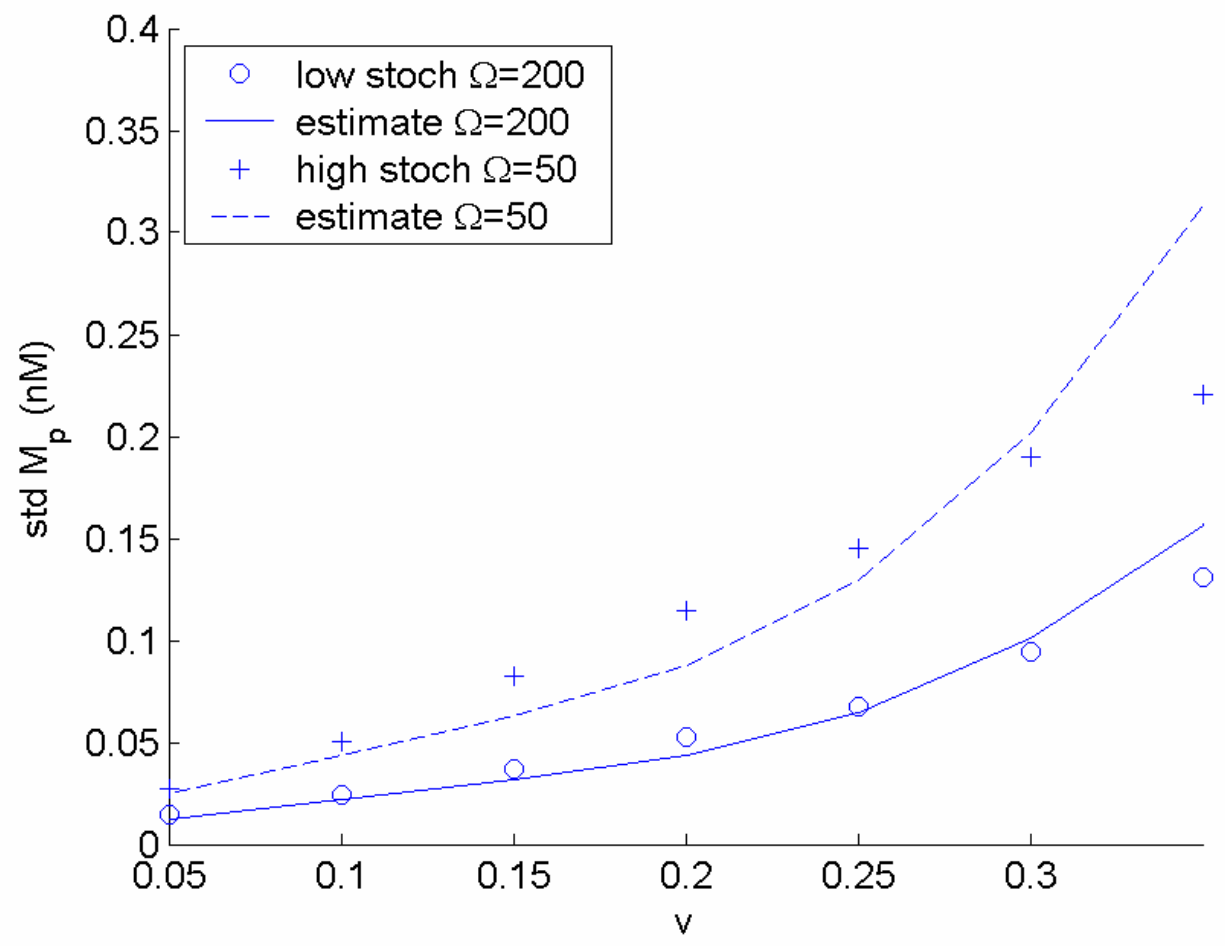

FIGURE 6 

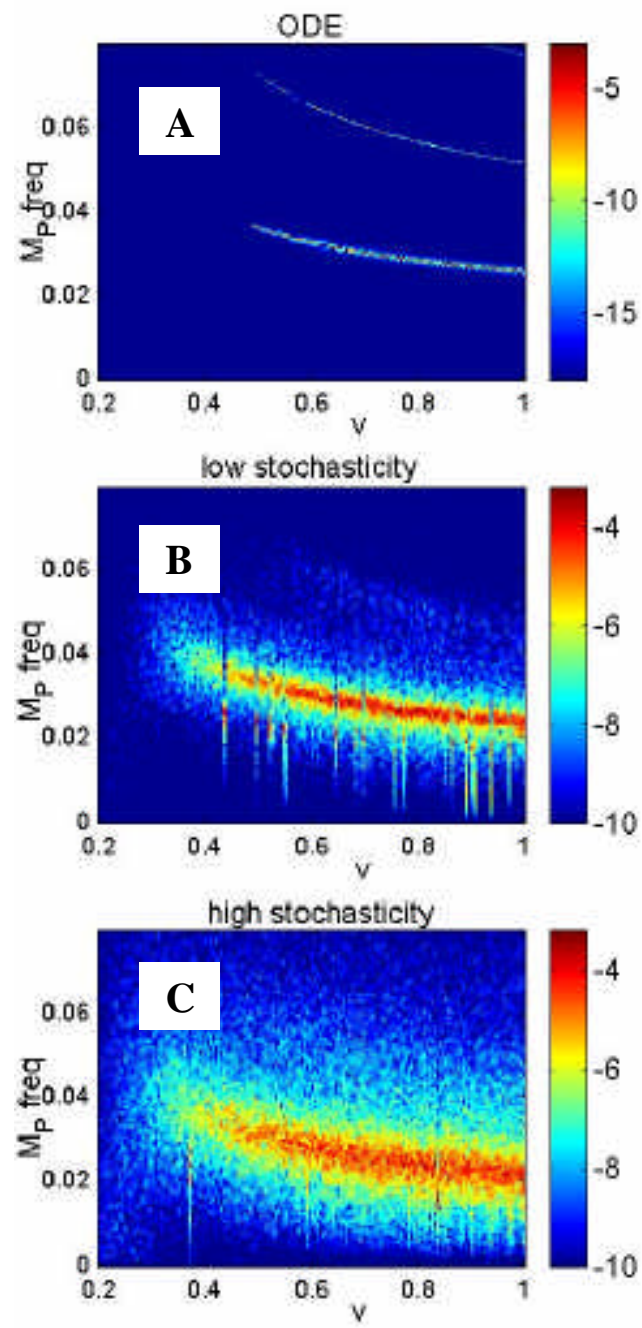

FIGURE 7 